\title{
Visual test determination of trace amounts of germanium in the form of an ionic associate of 12-molybdogermanate with astrafloxin
}

\author{
Tetiana Selivanova ${ }^{1, *}$, Andrey Vishnikin ${ }^{2}$, and Lyudmila Tsiganok $^{2}$ \\ ${ }^{1}$ Kryvyi Rih State Pedagogical University, Kryvyi Rih, Ukraine \\ ${ }^{2}$ Oles Honchar Dnipro National University, Dnipro, Ukraine
}

\begin{abstract}
Sorption-colorimetric and naked eye determination of germanium as ion associates of $\mathrm{GeMo}_{12} \mathrm{O}_{40}{ }^{4-}$ with triphenylmethane dyes is described. The sorption of the ionic associates (IA) of $12-$ molybdogermanat with astrafloxin (AF) on filter paper was studied. The colored scales for naked eye detection and dependence of chromaticity coordinates from the germanium (IV) concentration were obtained. The methods were applied to the determination of germanium (IV) in coked coal, iron ore, and in waters in the concentration range from $4 \cdot 10^{-8}$ to $1 \cdot 10^{-6} \mathrm{~mol} \cdot \mathrm{L}^{-1}$. The developed test-systems for the determination of Germanium in natural and technological objects were tested in the course of training students of chemistry of Kryvyi Rih State Pedagogical University.
\end{abstract}

\section{Introduction}

Control of the content of germanium is necessary when it is extracted from ores, concentrates, coal, in its production, in cosmetics and medicines, in food. Plants that are capable of absorbing germanium and its compounds from the soil include: ginseng, garlic, camphor, aloe, tomatoes, beans, chickpeas, sunflower seeds, mushrooms and wheat bran. Germanium is also found in milk and in salmon meat. Germanium is an important trace element for humans, capable of accumulation. But its excess in the body or lack leads to the formation of various diseases. Germanium deficiency can be dangerous, since in this case the risk of the onset and development of cancer, as well as osteoporosis, is increased.

Today it is necessary to have reliable, simple and relatively fast methods for its determination. The main methods for the determination of germanium (IV) today: atomic absorption method with electro-thermal atomization of the sample (AAS-ETA) [1, 2], with mandatory preliminary concentration, atomic emission spectroscopy with inductively coupled plasma (AESICP) [4], voltampermetric determination of germanium. Highly selective methods of atomic absorption analysis with electrothermal atomization or hydride version have their drawbacks. First of all, matrix elements interfere strongly with the determination, and the separation process only complicates and increases the analysis time. X-ray spectral and neutron activation methods to achieve sensitive, $\mathrm{Sn}=10^{-3} \mu \mathrm{g}$. But as for Germanium there is the problem of the formation of refractory carbides, introduced impurities from reagents, and other factors that interfere with the determination using physical methods of determination.

A significant part of the existing methods for determining germanium (IV) are spectrophotometric, with mandatory separation procedures. The main methods of separation are distillation or extraction of germanium chloride in a strongly acidic medium in the form of a complex with phenylfluorone or other reagents. But these methods also have their drawbacks the slow formation of a complex with phenylfluorone.

Therefore, it is relevant to develop highly sensitive, express methods for the determination of low contents of germanium (IV) and the creation of test methods for its semiquantitative determination.

To enhance the analytical signal in modern analysis, began to use the inclusion of chromophore groups with a high molar absorption coefficient in the analytical form. Until this time, triphenylmethane dyes, antipyrine and rhodamine dyes were most widely used (Table 1). The $\mathrm{pH}$ range in which these dyes exist in a cationic form is narrow. Recently, polymethine series dyes with a high molar absorption coefficient and a sufficiently wide $\mathrm{pH}$ range for the existence of singly charged cations have attracted their attention.

With these dyes, ionic associates (IA) of heteropolyanions (HPA) form solutions or pseudocolloidal solutions that allow the determination of heteroatom elements at a concentration level of $10^{-8}-10^{-7} \mathrm{~mol} / \mathrm{L}[3,6]$.

Currently, there are not enough test methods for determining germanium.

This work is devoted to studying the conditions of selective sorption of the ionic associate (IA) of the molybdogermanium heteropolyanion (GPA) with the

* Corresponding author: vitro090@gmail.com 
representative of polymethine dyes - astrafloxin (AF) on cellulose paper (Table 1).

\section{Reagents and equipment}

All solutions were prepared using bidistilled water and stored in plastic containers. To obtain the IA of the molybdogermanium complex (MGK), the following was used: the initial $0.01 \mathrm{M}$ solution of germanium (IV) was prepared by dissolving the corresponding portion of the $\mathrm{GeO}_{2}$ grade of os.h. with a solution $\mathrm{pH}$ of 11.5; $0.1 \mathrm{M}$ solution $\mathrm{Na}_{2} \mathrm{MoO}_{4}$ - from the recrystallized $\mathrm{Na}_{2} \mathrm{MoO}_{4} \times 2 \mathrm{H}_{2} \mathrm{O}$ grade of the chemical grade product recrystallized from a water-alcohol medium; $1 \cdot 10^{-3} \mathrm{M}$ solution of astrafloxine was prepared from the preparation of the analytical grade mark $\mathrm{C}_{25} \mathrm{H}_{29} \mathrm{~N}_{2} \mathrm{Cl}$ (Basic Red 12, 1,3,3-trimethyl-2- [3- (1,3,3-trimethyl-2indolinylidene ) propenyl] - 3H-indolium chloride). Solutions of a lower concentration were obtained by diluting the stock solutions. For dynamic sorption on filter paper, a column was used (Fig. 1):

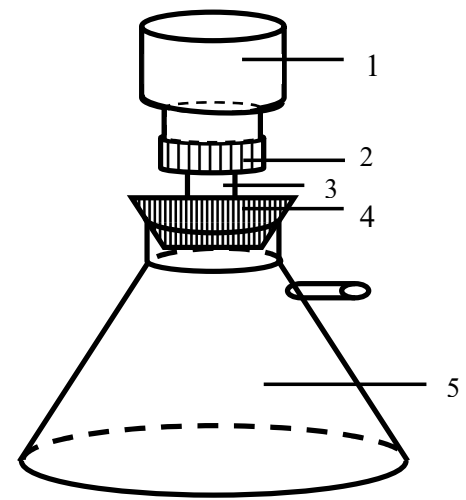

Fig. 1. Column for dynamic sorption on filter paper: $1-\mathrm{a}$ funnel with thread (2) and a plate for filter paper; 3 - glass connecting tube; 4 - rubber stopper; 5 - flask with a tube for filtering under vacuum.

Table 1. Photometric determination of Germanium in the form of IA with HPA with organic dyes.

\begin{tabular}{|c|c|l|l|c|c|c|}
\hline Element & Analytical form & \multicolumn{1}{|c|}{ Colorant } & $\lambda, \mathbf{n m}$ & $\mathbf{\varepsilon}, \mathbf{l} \cdot \mathbf{m o l e}^{\mathbf{- 1}} \cdot \mathbf{c m}^{\mathbf{- 1}}$ & detection limit, $\mathbf{~ m g} / \mathbf{l}$ & Source \\
\hline & & Crystalline violet & 590 & $1,8 \cdot 10^{-5}$ & $0,025-0,3$ & {$[1,3]$} \\
& & Malachite green & 620 & $1,6 \cdot 10^{-5}$ & $0,030-0,4$ & {$[6,3]$} \\
Germanium & \multirow{2}{*}{$\mathrm{GeMo}_{12} \mathrm{O}_{40}{ }^{4-}$} & Brillian green & 620 & $1,93 \cdot 10^{-5}$ & $0,025-0,35$ & {$[1,3]$} \\
& & Chrompyrazole & 610 & $0,43 \cdot 10^{-5}$ & $0,1-1,0$ & {$[5,8]$} \\
& & Rhodamine 6G & 610 & $1,34 \cdot 10^{-5}$ & $0,025-0,4$ & {$[6,11,10]$} \\
\hline
\end{tabular}

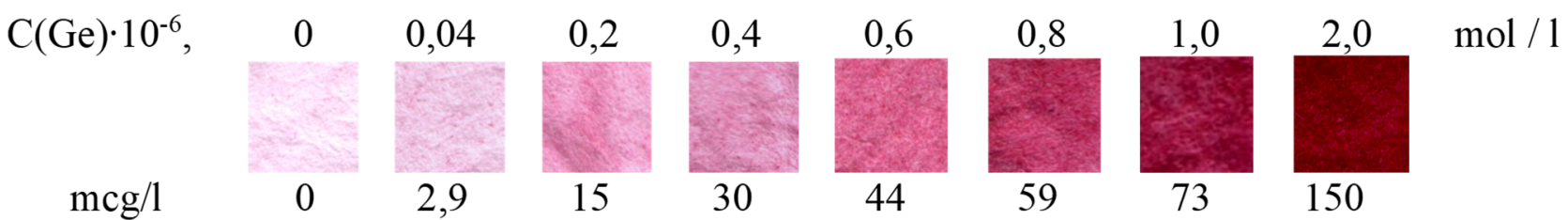

Fig. 2. Scanned test scale for visual test determination of $\mathrm{GeO}_{4}{ }^{2-}$ in the form of IA 12-MGK with astrafloxin on filter paper.

\section{Experiment techniques}

Preparation of $\beta-\mathrm{GeMo}_{12} \mathrm{O}_{40}{ }^{4-}$ IA with astrafloxin: the necessary volumes of $1 \cdot 10^{-6} \mathrm{M}$ Ge (IV) solution were added to $25 \mathrm{ml}$ flasks to obtain concentrations of $0,04 \cdot 10^{-6}-2 \cdot 10^{-6} \mathrm{~mol} / \mathrm{L}, 0$ each, $6 \mathrm{ml}$ of $1 \cdot 10^{-2} \mathrm{~mol} / \mathrm{L}$ $\mathrm{Na}_{2} \mathrm{MoO}_{4}$ solution, $0.5 \mathrm{~mol} / \mathrm{L} \mathrm{H}_{2} \mathrm{SO}_{4}$ solution to create the required $\mathrm{pH}(1.5-2.0)$. The reaction is fast. Then, 2 $\mathrm{ml}$ of a $0.6 \mathrm{M}$ solution of tartrate acid were added, after which the molybdogermanic acid solution was reacidified, $1.5 \mathrm{ml}$ of $5 \mathrm{M} \mathrm{H}_{2} \mathrm{SO}_{4}$. Then, in small portions, with constant stirring, $1 \mathrm{ml}$ of $10^{-4} \mathrm{~mol} / \mathrm{L} \mathrm{AF}$ was added, and distilled water was brought to the mark.

Obtaining a test scale for the visual test determination of $\mathrm{GeO}_{4}{ }^{2-}$ dynamic sorption was used. The resulting IA solution was passed through filter paper. Accelerated the passage of the solution using a vacuum pump. The resulting sample was removed from the column, dried in air, and then the resulting scale was scanned (Fig. 2).

In the absence of equipment for measuring diffuse reflection, colorimetric analysis is a simple and affordable method that is not inferior in efficiency. Measurement of staining intensity of IA sorbates on paper.

The method of carrying out colorimetric measurements: the scale prepared for the abovementioned method is scanned against a white background in the color image mode (separation ability $600 \times 600 \mathrm{dpi}$ or more) The brightness / contrast ratio is set to $30 / 0$. Using the Paint graphical editor, cut out $170 \times 170$ or smaller pixels from the scanned image. For each pixel in the image, R, G, B - color coordinates [2] are calculated and averaged over the entire data array.

\section{Results and discussion}

We studied the optimal conditions for the formation of a colored ionic asociate (IA) of a 12-molybdogermanium complex (12-MGC) with polymethine dye cations astrafloxin (AF) in a solution and its sorption on cellulose paper. The formation of the ionic associate AF-MGK was carried out in two stages.

At the first stage, a yellow oxidized molybdogermanium complex was obtained by 
acidification of a mixture of solutions that contain $\mathrm{GeO}_{3}{ }^{2-}$ and $\mathrm{MoO}_{4}{ }^{2-}$.

The second acidification was carried out to prevent the formation of persistent IA Astrafloxin with isopolymolybdate ions. The excess of molybdate ions was masked with hydroxy acids.

The reaction of the formation of IA MGK with astrafloxin occurs instantly. The reaction is selective with a fairly high sensitivity. The selectivity of the formation reaction may be impaired by the formation of colored IAs with large anions, but their solubility is much greater than the solubility of IAs with heteropolyanions. Therefore, there is no interfering effect of such anions: $\mathrm{HCO}_{3}{ }^{-}, \mathrm{SO}_{4}{ }^{2-}, \mathrm{NO}_{3}{ }^{-}$. Table 2 shows data on the effect of a number of ions on the determination of germinate ions.

Table 2. The maximum excess of foreign ions that does not affect the determination of Ge ((IV) in the form of $\mathrm{GeMo}{ }_{12} \mathrm{O}_{40}{ }^{4-}$ with AF. $\mathrm{C}(\mathrm{Ge})=4 \cdot 10^{-7} \mathrm{~mol} / \mathrm{L}$.

\begin{tabular}{|c|c|c|c|c|c|c|}
\hline interfering ion & $\begin{array}{c}\mathbf{N a}^{+}, \\
\mathbf{K}^{+}, \\
\mathbf{M g}^{2+}\end{array}$ & $\begin{array}{c}\mathrm{NO}_{3}^{-} \\
\mathrm{Cl}^{-}, \\
\mathrm{AsO}_{4}{ }^{3-}\end{array}$ & \begin{tabular}{|l|}
$\mathbf{N i}^{2+}$ \\
$\mathrm{Zn}^{2+}$ \\
$\mathrm{Cu}^{2+}$
\end{tabular} & $\mathrm{Fe}^{3+}$ & $\begin{array}{l}\mathrm{CO}_{3}{ }^{2-}, \\
\mathrm{PO}_{4}{ }^{3-}\end{array}$ & $\mathrm{SiO}_{3}{ }^{2-}$ \\
\hline $\begin{array}{c}\text { molar ratio } \\
\mathrm{C}_{\mathrm{Ge}}: \mathrm{C}_{\mathrm{X}}\end{array}$ & $1: 1000$ & $1: 500$ & $1: 400$ & $1: 100$ & $1: 50$ & $1: 5$ \\
\hline
\end{tabular}

The decrease in the interfering effect of silicates in the determination of germanate ions is explained by the fact that the reaction for the formation of MGC proceeds in a more acidic environment. In this $\mathrm{pH}$ range, molybdosilicate is not fully formed. In this case, the molybdosilicate complex (MSC) is formed very slowly for 15-20 minutes, and the modibdogermanate complex - quickly, 1-2 minutes.

We have for the first time shown the possibility of a highly sensitive visual test determination of Ge (IV) after selective concentration of IA 12-MGA with polymethine dye AF.

Calibration graphs were obtained for the colorimetric determination of Ge (IV) in the coordinates of the $\mathrm{R}$ and $\mathrm{B}$ functions (red and blue) $-\log \mathrm{C}$ (Table 3 ) or $(255-G)^{2} / 2 G=f(C)$ and the possibility of correlation of this dependences with spectrophotometric in coordinates A - $(255-\mathrm{G})^{2} / 2 \mathrm{G}$.

Table 3. Color coordinates R, G, B obtained after scanning stained samples of filter paper with sorbed IA $\mathrm{GeMo}_{12} \mathrm{O}_{40}{ }^{4-}-\mathrm{AF}$

\begin{tabular}{|c|c|c|c|c|}
\hline $\mathbf{C}_{\mathrm{Ge}} \cdot \mathbf{1 0}^{\mathbf{- 6}}, \mathbf{m o l} / \mathbf{L}$ & $\mathbf{R}$ Color & G Color & B Color & $\lg (\mathbf{C})$ \\
\hline blank test & 243 & 242 & 241 & 0 \\
\hline $\mathbf{0 , 0 4}$ & 230 & 196 & 186 & $-7,398$ \\
\hline $\mathbf{0 , 2}$ & 188 & 177 & 127 & $-6,699$ \\
\hline $\mathbf{0 , 4}$ & 154 & 169 & 99 & $-6,398$ \\
\hline $\mathbf{0 , 6}$ & 135 & 134 & 68 & $-6,222$ \\
\hline $\mathbf{0 , 8}$ & 127 & 87 & 39 & $-6,097$ \\
\hline $\mathbf{1}$ & 113 & 79 & 24 & $-6,00$ \\
\hline $\mathbf{2}$ & 92 & 44 & 12 & $-5,699$ \\
\hline
\end{tabular}

The dependence of the R, G, and B color coordinates on the concentration of IA is exponential (Fig. 3.a, Fig. 3.b). $\mathrm{R}$ and $\mathrm{G}$ color coordinates upon IA $\mathrm{GeMo}_{12} \mathrm{O}_{40}{ }^{4}$ - AF sorption change most naturally and in a wider interval. Data were processed using the modified Kubelka-Munk equation (Fig. 4). The equation of the calibration graph in coordinates $(255-G)^{2} / 2 G=f(C)$, where $G$ is the value of the color coordinate $\mathrm{R}$, and $\mathrm{C}$ is the concentration of Ge (IV) in $\mathrm{mol} / \mathrm{L}$, is described by the expression $(-4,1 \pm 1,5)+$ $(3,16 \pm 0,11) \cdot 108 \cdot \mathrm{C}$ Ge $(\mathrm{IV})$, the correlation coefficient is 0.995 . The detection limit calculated by the $3 \mathrm{Sa} / \mathrm{b}$ formula is $4 \cdot 10^{-8} \mathrm{~mol} / \mathrm{L}$.

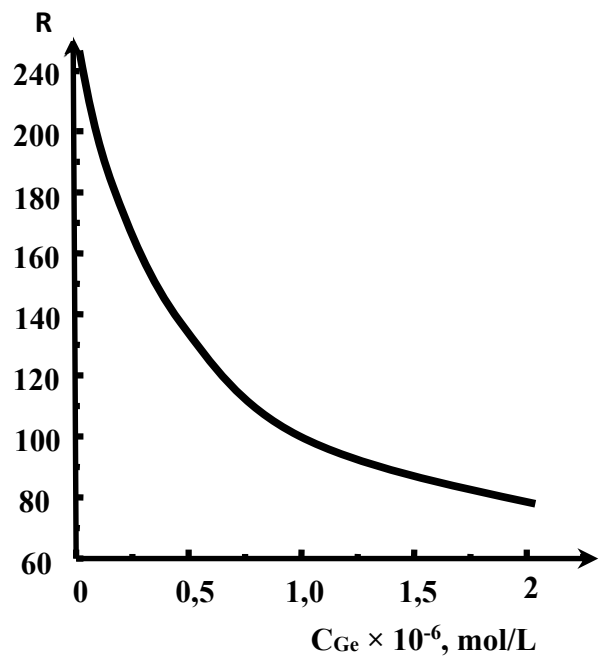

Fig. 3a. Dependence of coordinates R - functions of Ge (IV) concentration after sorption $\mathrm{GeMo}_{12} \mathrm{O}_{40}{ }^{4-}$ - AF

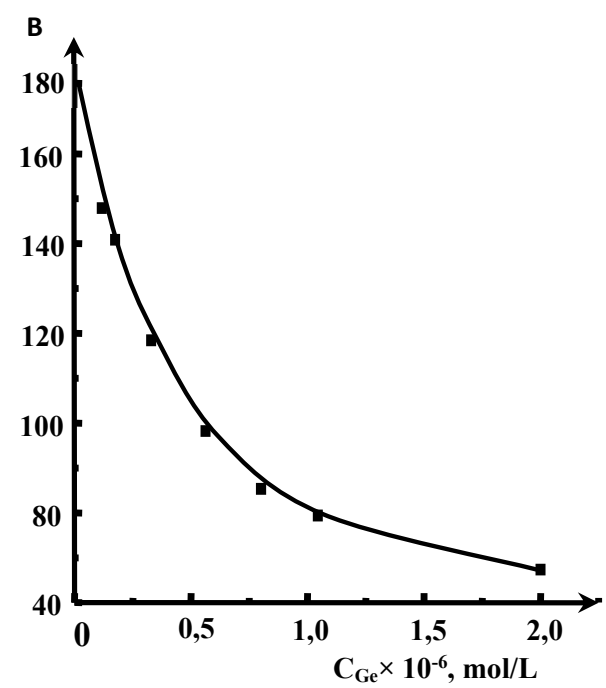

Fig. 3.b. Dependence of coordinates B - functions of Ge (IV) concentration after sorption IA $\mathrm{GeMo}_{12} \mathrm{O}_{40}{ }^{4-}$ - AF.

Calibration graphs were obtained for the colorimetric determination of Ge (IV) in the coordinates of the $\mathrm{R}$ and $\mathrm{B}$ functions (red and blue) $-\log \mathrm{C}$ (Table 3 ) or $(255-\mathrm{G})^{2} / 2 \mathrm{G}=\mathrm{f}(\mathrm{C})$ and the possibility of correlation of this dependences with spectrophotometric in coordinates A - $(255-\mathrm{G})^{2} / 2 \mathrm{G}$.

The need to analyze many different objects for the content of germanium requires the development of simple sensitive methods for a preliminary assessment of their content for the correct further selection of quantitative methods.

The developed method for the determination of germanium in the form of AF-12-MGK IA was used and 
tested in the analysis of coking coal and iron ore. For analysis, we took coal from the coke-chemical plant in Kryvyi Rih, and iron ore from the Krivorozhskoye deposit. In coke-chemical production, the bulk of germanium remains in coke and only a small amount goes into tar water and tar. Coking coal is one of the sources of germanium production. The correctness of the results was monitored by the standard method (Table 4) using phenylfluorone. Also, the method of verification of model solutions (Table 5).

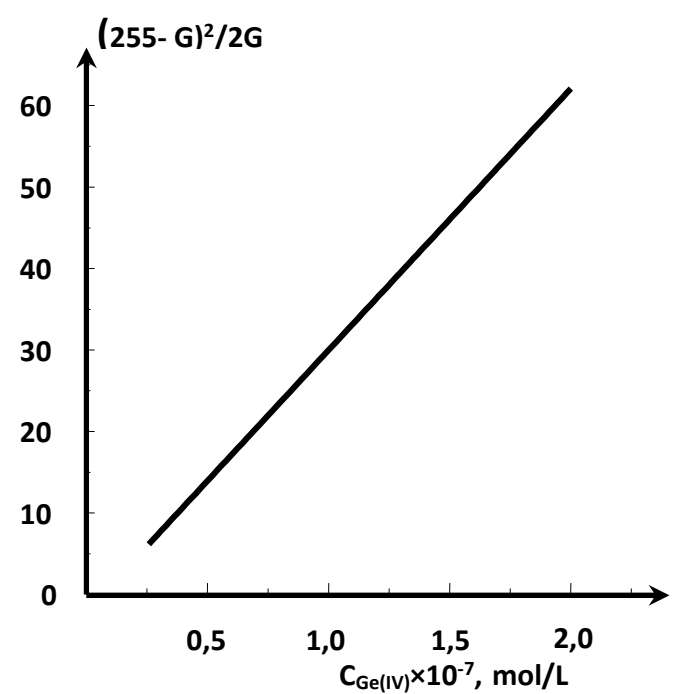

Fig. 4. Calibration chart for colorimetric determination of Ge (IV) in the form of IA KF with 12-MGK. Dependence of the modified Kubelka-Munk function on the concentration of germanium (IV).

Table 4. The results of the determination of Ge (IV),

$$
\alpha=0.95, n=6
$$

\begin{tabular}{|c|c|c|}
\hline $\begin{array}{c}\text { object of } \\
\text { analysis }\end{array}$ & $\begin{array}{c}\text { colorimetric } \\
\text { determination of IA } \\
\text { AF with 12-MGK, } \\
\text { discovered Ge } \\
\left(\mathbf{X}_{\mathbf{c p} \pm \Delta)} \mathbf{\%}\left(\mathbf{S}_{\mathbf{r}}\right)\right.\end{array}$ & $\begin{array}{c}\text { Standard determination } \\
\text { procedure using } \\
\text { phenylfluorone, } \\
\text { discovered Ge }\left(\mathbf{X}_{\mathbf{c p}} \pm \Delta\right), \\
\mathbf{\%}\left(\mathbf{S}_{\mathbf{r}}\right)\end{array}$ \\
\hline $\begin{array}{c}\text { coking } \\
\text { coal }\end{array}$ & $(2 \pm 0,08) \cdot 10^{-6}(0,03)$ & $(1,67 \pm 0,06) \cdot 10^{-6}(0,04)$ \\
\hline iron ore & $(1 \pm 0,08) \cdot 10^{-6}(0,04)$ & $(1,25 \pm 0,06) \cdot 10^{-6}(0,04)$ \\
\hline
\end{tabular}

Table 5. Results of visual-test determination of $\mathrm{GeO}_{3}{ }^{2-}$ in model solutions by the introduced-found method

$$
(P=0,95, n=6)
$$

\begin{tabular}{|c|c|c|c|c|c|}
\hline sample & \multirow{2}{*}{$\begin{array}{c}\text { Introduced } \\
\mathbf{G e}^{\mathbf{I V}}, \boldsymbol{\mu g} / \mathbf{l}\end{array}$} & $\begin{array}{c}|c| \\
\text { propcovered } \\
\text { method }\end{array}$ & $\mathrm{S}_{r}$ & $\begin{array}{c}\text { Method with } \\
\text { phenylfluorone }\end{array}$ & $\mathrm{S}_{r}$ \\
\hline $\begin{array}{c}\text { drinking } \\
\text { water } \\
\text { "Bon } \\
\text { Boisson" }\end{array}$ & 58,0 & $59,4 \pm 2,5$ & 0,03 & $58,5 \pm 2,0$ & 0,03 \\
\hline $\begin{array}{c}\text { distilled } \\
\text { water }\end{array}$ & 14,5 & $14,7 \pm 0,14$ & 0,04 & $14,6 \pm 0,14$ & 0,03 \\
\hline
\end{tabular}

Thus, the possibility of using colorimetry in the analysis is confirmed, but not as a substitute for spectrophotometry, but as an element of preanalysis. In spectrophotometry, integrated spectral characteristics are measured, in colorimetry, the components of color, that is, the color is differentiated into components. These are the so-called primary color coordinates: red (R), blue (B) and green $(\mathrm{G})$ [13].

Calculations of these coordinates, their relationship with the concentration of the colored substance can increase the sensitivity of the determination, recognize shades, find steps for varying the concentrations in the manufacture of visual test scales.

Colorimetry, as a method of objective color measurement with computer processing of data, is widely used in industry: printing, varnishing, textiles, food, pharmaceuticals, and also began to be used in analysis.

At the moment, colorimetry is distinguished as an independent physicochemical research method. Colorimetry can be a powerful tool in material science, visual express analysis. In the literature they give recommendations for constructing color scales. There is an opinion that the minimum step of the scale corresponds to the Golden Ratio (Fibonacci number), the use of which minimizes the error of analysis [14].

Compared to the standard spectrophotometric method using phenylfluorone, the test method using astrafloxin has several advantages:

- is the lower definition threshold

- speed of determination

- greater availability of materials and reagents

- ability to use for on-site analysis

- no need to use expensive equipment.

At the same time, the disadvantages of the test method are:

- less reliability

- the method is semi-quantitative.

Thus, the test method cannot claim to be used in precision research, but can be used for educational research and approximate content assessments of Germanium.

The use of test systems in onsite analysis as part of the training course is one of the elements of sustainable development in learning.

Students gain an understanding the ability to determine micro-quantities Germanium is an important aspect of the eco-chemical assessment of the environmental impact of industrial enterprises. of chemistry for sustainable development as a new approach to the planning and implementation of chemical reactions and chemical processes. He learns that at the planning stage of the experiment, an assessment is needed not only of the target chemical properties of the future product, but also an assessment of environmental risks.

Currently, this method of the semiquantitative determination of germanium is used in laboratory and chemical practice at the Kryvyi Rih State Pedagogical University. As part of the analysis in place. The experience of use showed the simplicity and accessibility of performing operations to prepare a test scale and subsequent colorimetric analysis.

During the chemical training students studied the water of various reservoirs (including man-made ones) 
of the city of Kryvyi Rih and surrounding areas. The study was conducted on many parameters, including the definition of Germanium. Germanium is a potentially dangerous element for human health [15].

However, the results of research over the last 5 years show that the content of Germanium, even in man-made reservoirs, does not exceed $1 \mu \mathrm{g} / \mathrm{l}$. This is due to the rather low mobility of inorganic compounds in Germanium. It also shows that there is no potential environmental threat from Germany in such an industrial region as the city of Kryvyi Rih.

\section{References}

1. A.M. García-Campaña, F. Alés Barrero, A. Lupiáñez González, M. Román Ceba. Analytica Chimica Acta. 1-2 (447), 219-228 (2001). doi:10.1016/S0003-2670(01)01288-0.

2. H. Matusiewicz, M. Krawczyk. Anal. Lett. 16(46), 2543 (2010). doi:10.1080/00032711003725631

3. I.P. Alimarin, Zhurn. analit. himii 6(39), 965(1984)

4. A.B. Vishnikin, Dissertation, Odessa, 2012

5. L.I. Ganago, Zhurn. analit. himii 26(1), 104-109 (1971)

6. E.N. Dorohova, Zhurn. analit. himii 8(50), 870 (1995)

7. F.V. Mirzoyan, Zhurn. analit. himii 3(55), 289 (2000)

8. F.V. Mirzoyan, Talanta. 27, 1050 (1980)

9. L.V. Myishlyaeva, Nauka, 209 (1972)

10. M. McMahon, F. Regan, H. Hughes, Food Chemistry 3(97), 411 (2006). doi:10.1016/j.foodchem.2005.05.018

11. S.K. Tobia, M.F. El-Shahat, E.A. Saad, Microchemical Journal 23(4), 525-529 (1978)

12. A. Harada, T. Tarutani, K. Yoshimura, Analytica Chimica Acta 209, 333-338 (1988)

13. V.M. Ivanov, O.V. Kuznetsova, Uspekhi khimii. 5 (70), 411 (2001). doi:10.1070/RC2001v070n05ABEH000636

14. S.V. Himchenko, L.P. Eksperiandova, Tsvetometriya $v$ instrumentalnom $i$ vizuanom test analize (Chromaticity in instrumental and visual test analysis) (Lambert Academic Publishing, 2014)

15. Shyy-Hwa Tao, P.M. Bolger, Regulatory Toxicology and Pharmacology 25(3), 211-219 (1997). doi:10.1006/rtph.1997.1098 